\title{
EFEITO DAS ATIVIDADES DE ECOTURISMO SOBRE A RIQUEZA E A ABUNDÂNCIA DE ESPÉCIES DE MAMÍFEROS DE MÉDIO E GRANDE PORTE NA REGIÃO DO CRISTALINO, MATO GROSSO, BRASIL ${ }^{1}$
}

\author{
Ednaldo Cândido Rocha ${ }^{2}$, Elias Silvaª ${ }^{3}$ Julio Cesar Dalponte e Gisele Mendes Lessa del Giúdice $^{5}$
}

RESUMO - Este estudo foi desenvolvido nas Reservas Particulares do Patrimônio Natural Cristalino (670 ha) e Lote Cristalino (6.539 ha) e no Parque Estadual Cristalino (184.900 ha). Essas unidades de conservação são contíguas e estão localizadas no extremo centro-norte do estado do Mato Grosso, em locais considerados prioritários para a conservação, em função da alta biodiversidade e endemismos, além da elevada pressão antrópica. Desta forma, este trabalho objetivou conhecer, avaliar e comparar a estrutura das populações de mamíferos de médio e grande porte, em termos de riqueza e abundância das espécies em ambientes sem turismo e com atividades de ecoturismo na região do Cristalino. Para tanto, no período compreendido entre maio de 2008 a fevereiro de 2010 foram amostrados ambientes com floresta primária, os quais apresentavam dois níveis de perturbação antrópica: sem turismo e com atividades de ecoturismo. Os dados foram coletados utilizando a amostragem de distâncias em transecções lineares, que totalizou 468,3 Km percorridos nos períodos diurno e noturno, e registro de pegadas em parcelas previamente preparadas ( $\mathrm{n}=660$ parcelas vistoriadas), além de percursos fluviais no rio Cristalino e buscas aleatórias nos locais onde não ocorriam caminhos. Registros de 37 espécies de mamíferos foram obtidos, sendo 33 de médio e grande porte e quatro de pequeno porte. Não houve diferença estatisticamente significativa na riqueza de espécies dos ambientes sem turismo e com ecoturismo, sendo que a similaridade de espécies entre eles foi alta (88\%). No entanto, três táxons apresentaram abundância inferior nos ambientes com turismo: Dasyprocta leporina (Linnaeus, 1766), Mazama spp. e Dasypus kappleri Krauss, 1862. Percebe-se, portanto, que o impacto negativo das atividades de ecoturismo desenvolvidas na área de estudo foi de pequena magnitude, em termos de riqueza e abundância de mamíferos de médio e grande porte. Assim, empreendimentos de ecoturismo se apresentam como importante atividade econômica a ser desenvolvida em áreas com potencial turístico na Amazônia.

Palavras-chave: Mastofauna, Unidade de Conservação e Amazônia Meridional.

\section{EFFECT OF ECOTOURISM ACTIVITIES ON RICHNESS AND ABUNDANCE OF SPECIES OF MEDIUM AND LARGE MAMMALS IN THE CRISTALINO REGION, MATO GROSSO, BRAZIL}

\begin{abstract}
This study was carried out in the Private Natural Heritage Reserves Lote Cristalino (670 ha), Cristalino (6,539 ha) and in Cristalino State Park (184,900 ha). These protected areas are contiguous and are located in the extreme north-central State of Mato Grosso, in areas considered priorities for conservation due to the high biodiversity and endemism and the high human pressure. Thus, this study focused to evaluate and compare the structure of populations of medium and large mammals in terms of richness and abundance in environments without tourism and with ecotourism activities in Cristalino region. Therefore, in the period from May 2008 to February 2010, primary forest environments with two levels of anthropogenic disturbance were sampled: no tourism at all and ecotourism activities. Data were collected by using the distance samplings
\end{abstract}

\footnotetext{
${ }^{1}$ Recebido em 31.01.2012 aceito para publicação em 04.06.2012

${ }^{2}$ Universidade Estadual de Goiás, UnU de Ipameri. E-mail: <ednaldorocha@yahoo.com.br>.

${ }^{3}$ Universidade Federal de Viçosa, UFV, Brasil. Departamento de Engenharia Florestal. E-mail: <eshamir@ufv.br>.

${ }^{4}$ Rastos - Planejamento \& Serviços Ambientais para Vida Silvestre. E-mail: <jcdalponte@ hotmail.com>.

${ }^{5}$ Universidade Federal de Viçosa, Centro de Ciências Biológicas e da Saúde. E-mail: <gislessa@ yahoo.com.br>.
} 
in linear transect, totalizing 468,3 kilometers traveled during daytime and nighttime, and the record of footprints in previously prepared plots ( $n=660$ surveyed plots), in addition to river corridors in Cristalino River and random searches in places with difficult access due to the lack of roads. Records of 37 species of mammals were obtained, which 33 were medium and large size and four small ones. There was no statistically significant difference in species richness of the environments without tourism and with ecotourism, and the similarity of species among them was quite high (88\%). However, three taxa were less abundant in environments with tourism: Dasyprocta leporina (Linnaeus, 1766), Mazama spp. and Dasypus kappleri Krauss, 1862. Therefore, it can be observed that the negative impact of ecotourism activities developed in the study area presented a small magnitude, in terms of richness and abundance of medium and large mammals. Thus, ecotourism ventures are presented as important economic activity to be developed in areas with touristic potential in the Amazon.

Keywords: Mammals fauna, Protected areas and Southern Amazon.

\section{INTRODUÇÃO}

Cerca de 700 espécies de mamíferos ocorrem em território brasileiro (PAGLIA et al., 2012), o que representa aproximadamente $12 \%$ da mastofauna do mundo. Esses números fazem que o Brasil seja o país mais diverso do planeta no grupo dos mamíferos (COSTA et al., 2005). A Amazônia é o bioma brasileiro com a maior riqueza de mamíferos, contando com aproximadamente $60 \%$ das espécies que ocorrem no país, além de apresentar grau de endemismo elevado, com cerca de 230 espécies de mamíferos ocorrendo exclusivamente nesse bioma (PAGLIA et al., 2012). A maioria das espécies de vertebrados terrestres da Amazônia não está distribuída homogeneamente ao longo de todo o bioma, mas em regiões claramente delimitadas pelos grandes rios, as quais são chamadas de "áreas de endemismo" (SILVA et al., 2005).

A região de Alta Floresta, extremo Norte do Estado do Mato Grosso, foi considerada uma das áreas prioritárias para a conservação da Amazônia Meridional, em razão da alta diversidade e de endemismos, além de estar sob grande pressão antrópica (BRASIL, 2007). As Reservas Particulares do Patrimônio Natural (RPPNs) Cristalino e Lote Cristalino e o Parque Estadual Cristalino, localizadas no extremo Norte do Estado do Mato Grosso, abrigam áreas ricas em biodiversidade, quando se considera a Amazônia Meridional brasileira (FEMA, 2002). Por sua localização estratégica, essas unidades de conservação integram o corredor ecológico meridional de conservação da Amazônia, constituindo importante barreira para conter o avanço do antropismo advindo da fronteira do "arco do desmatamento da Amazônia" no Norte do Estado do Mato Grosso (ZAPPI et al., 2011). Mas estudos sobre mamíferos nessas áreas ainda são incipientes (MICHALSKI; PERES, 2007)e, em sua maioria, constituemse de trabalhos não publicados.
No cenário atual, fica evidente que o processo de ocupação da Amazônia precisa ser pautado num modelo de desenvolvimento que minimize os impactos ambientais negativos e que busque a sustentabilidade dos recursos naturais. Nesse sentido, os empreendimentos de ecoturismo apresentam-se como alternativa para compatibilizar atividade econômica e conservação ambiental. Entretanto, é importante a realização de diagnósticos e monitoramentos ambientais para nortear o bom planejamento das atividades antrópicas, levando em consideração as potencialidades e as limitações do ambiente (LOBO; SIMÕES, 2010).

Nesses termos, o objetivo deste estudo foi avaliar e comparar a riqueza, a similaridade e a abundância de espécies de mamíferos de médio e de grande porte em dois tipos de ambientes terrestres na região do Cristalino, sendo eles: 1) ambientes sem uso antrópico e 2) ambientes utilizados em atividades de ecoturismo.

\section{MATERIAL E MÉTODOS}

\subsection{Caracterização da área de estudo}

O estudo foi conduzido simultaneamente em cinco unidades de conservação contíguas: as Reservas Particulares do Patrimônio Natural Cristalino I, II, III (aqui denominadas RPPNs Cristalino - 6.539 ha) e Lote Cristalino (670 ha) e o Parque Estadual Cristalino (184.900 ha). Esse bloco possui área de 192.109 ha, abrangendo os Municípios de Alta Floresta e Novo Mundo, no extremo Centro-Norte do Estado do Mato Grosso (09 $33^{\prime} 15^{\prime \prime} \mathrm{S}$ e $55^{\circ} 53^{\prime} 25^{\prime \prime} \mathrm{O}$ - coordenadas registradas no ponto central da área amostrada) (Figura 1).

As RPPNs abrigam o Hotel de Selva Cristalino (Cristalino Jungle Lodge), um empreendimento de ecoturismo, bem como as trilhas e demais estruturas

Revista Árvore, Viçosa-MG, v.36, n.6, p.1061-1072, 2012 


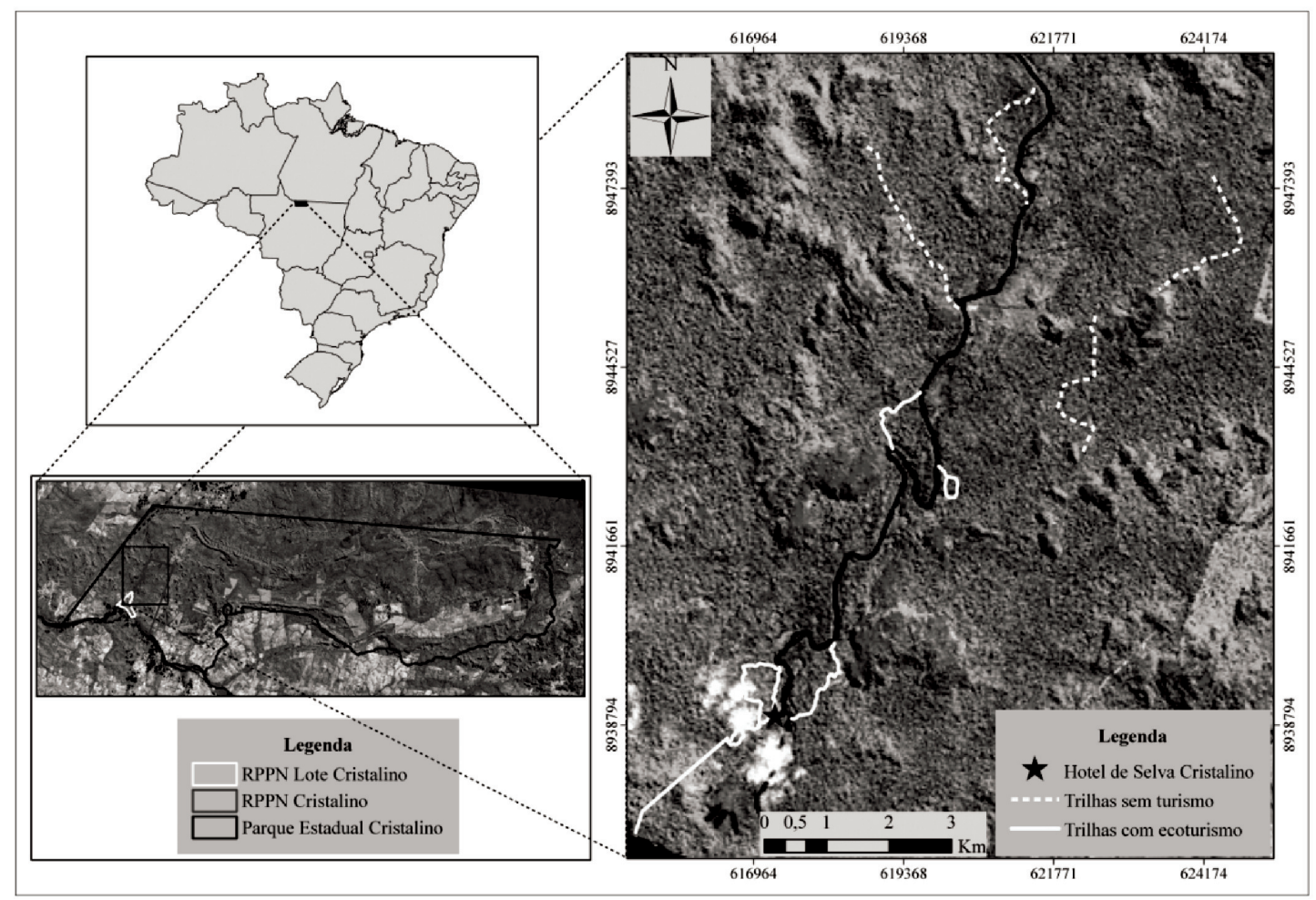

Figura 1 - Localização do Parque Estadual Cristalino e das RPPNs Cristalino e Lote Cristalino, estado do Mato Grosso, Brasil, evidenciando as trilhas amostradas nos ambientes sem turismo (traço branco pontilhado) e nos ambientes com ecoturismo (traço branco contínuo).

Figure 1 - Location of the Cristalino State Park and Private Reserves of Cristalino and Lote Cristalino, Mato Grosso state, Brazil, showing tracks sampled in environments without tourism (dotted white line) and in environments with ecotourism (continuous white line).

de apoio à visitação. A capacidade máxima das acomodações do hotel era de 50 pessoas, sendo rara a sua completa lotação. A demanda de visitação foi sazonal, sendo mais intensa entre junho e outubro, período em que o hotel chegou a abrigar cerca de 35 hóspedes/dia, concentrando aproximadamente $65 \%$ do total de turistas que visitaram a área. Durante este estudo, o Hotel de Selva Cristalino recebeu cerca de 950 visitantes/ano e, para a maior parte das atividades, o tamanho dos grupos que percorreram as trilhas limitouse a no máximo oito pessoas.

O termo "região do Cristalino" é usualmente utilizado para referir-se à porção mato-grossense da bacia do rio Cristalino, afluente do rio Teles Pires, que nasce na Serra do Cachimbo, no Sul do Estado do Pará, incluindo também parte da bacia do rio Nhandu, outro afluente do Teles Pires (ZAPPI et al., 2011).
O clima na região é considerado quente e úmido, com temperaturas anuais médias mínimas de $21^{\circ} \mathrm{C}$ e máximas de $30,5^{\circ} \mathrm{C}$. A pluviosidade média anual é de cerca de $2.000 \mathrm{~mm}$, mas apresenta uma estação seca definida, de maio a setembro, quando as médias mensais de precipitação são inferiores a 100 mm (NIMER, 1989; SEPLAN, 2001). A altitude varia entre 100 e $400 \mathrm{~m}$, crescentes do norte para o sul (ZAPPI et al., 2011).

A área de estudo está situada na interface entre a Amazônia e o Cerrado e, em termos de fisionomia, a vegetação apresenta características comuns a ambos os biomas, mas, em termos florísticos, a vegetação é quase que exclusivamente amazônica (ZAPPI et al., 2011). Segundo esses autores, no Parque Estadual Cristalino são encontradas florestas altas, densas, variando de perenifólias a completamente decíduas, floresta periodicamente inundada, mata de cipó aberta, 
vários tipos de campina/campinarana, vegetação associada a afloramentos rochosos, vegetação ribeirinha e lacustre. Um dos tipos florestais mais importantes é a floresta ombrófila densa, geralmente associada a solos argilosos (ZAPPI et al., 2011).

\subsection{Coleta dos dados}

A coleta dos dados foi conduzida entre maio de 2008 e fevereiro de 2010, em ambientes com dois diferentes níveis de utilização antrópica:

1 - Ambientes sem turismo - foram delineados e amostrados quatro transectos com comprimento de 2,82 a 3,25 km, dispostos em ambientes sem utilização antrópica para a realização dos levantamentos, os quais tinham, pelo menos, $1 \mathrm{~km}$ de afastamento entre eles (Figura 1).

2 - Ambientes utilizados em atividades de ecoturismo - levantamentos foram conduzidos ao longo de seis transectos na área, os quais também eram utilizados em atividades de ecoturismo nas RPPNs Cristalino e Lote Cristalino e no Parque Estadual Cristalino (Figura 1). As trilhas apresentaram comprimento entre 1,13 e 2,66 km e distavam entre 0,2 e $5 \mathrm{~km}$ do Hotel de Selva Cristalino (Figura 1). As trilhas eram utilizadas por no máximo dois grupos pequenos de pessoas percorrendo as trilhas no mesmo dia, em turnos diferentes.

Os transectos estavam posicionados em áreas com vegetação primária, predominando a floresta do tipo ombrófila densa em terra firme. Em menor escala, também foram incluídas áreas com Floresta Estacional Semidecidual e áreas com manchas de tabocais, presentes apenas em ambientes com ecoturismo.

Os levantamentos, censos por transecção linear (BUCKLAND et al., 1993; CULLEN JR.; RUDRAN, 2003), consistiram de percursos realizados durante caminhadas nos transectos, individuais ou em dupla, numa velocidade média de $1,33 \mathrm{~km} / \mathrm{h}$ (durante o dia a velocidade média foi de $1,47 \mathrm{~km} / \mathrm{h}$ e durante a noite, de $1,00 \mathrm{~km} / \mathrm{h}$ ), para a visualização de mamíferos. Os levantamentos foram realizados ao longo do dia (entre $06 \mathrm{~h} 00$ e $10 \mathrm{~h} 00$ e entre $14 \mathrm{~h} 30$ e $17 \mathrm{~h} 30$ ) e durante a noite (entre $18 \mathrm{~h} 30$ e $22 \mathrm{~h} 30$ e entre $01 \mathrm{~h} 30$ e $05 \mathrm{~h} 30$ ). Os esforços de amostragem nos transectos estão sintetizados na Tabela 1. Percursos de retorno nos transectos realizados no mesmo turno, após o término dos levantamentos, não foram considerados no cálculo da abundância.
Registros visuais e vocais dos mamíferos obtidos durante os levantamentos eram identificados e anotados, assim como a distância perpendicular entre o animal e a trilha, a identificação do transecto, a quantidade de indivíduos em cada grupo (no caso de animais gregários), a caracterização do ambiente amostrado, o horário dos levantamentos e das detecções, a posição dos animais na trilha, as condições climáticas e aspectos comportamentais relevantes.

Entre novembro de 2008 e fevereiro de 2010, oito transectos, sendo quatro nos ambientes sem turismo e quatro nos ambientes com ecoturismo, receberam 10 parcelas de 1,0 x 1,0 m cada para o registro de pegadas, instaladas ao longo das trilhas, com a distância aproximada de $100 \mathrm{~m}$ entre elas (PRADO et al., 2008). As parcelas foram preparadas revolvendo o substrato do próprio transecto, com o auxílio de enxadão e rastelo. As vistorias ocorreram cerca de 24 h após o preparo das parcelas, momento em que as pegadas eram registradas e apagadas, deixando as parcelas novamente preparadas para a vistoria do dia seguinte. Esse método, que foi utilizado para complementar o inventário das espécies, totalizou 310 parcelas vistoriadas nos ambientes sem turismo e 350 nos ambientes com ecoturismo.

Adicionalmente, detecções de mamíferos obtidas no retorno de levantamentos, fora dos transectos e das parcelas, em percursos fluviais no rio Cristalino e em buscas aleatórias nos locais onde não havia caminhos, também foram anotadas e consideradas para complementar o inventário das espécies da área estudada.

Neste estudo, seguiu-se a classificação taxonômica proposta por Wilson e Reeder (2005), exceto para a Ordem Primates, cuja nomenclatura seguiu Reis et al. (2008). Consideraram-se como mamíferos de médio e de grande porte aqueles cuja massa corporal ultrapassava $1 \mathrm{~kg}$ quando adultos, seguindo o critério adotado por Chiarello (2000) e Rocha e Silva (2009).

$\mathrm{Na}$ área de estudo havia ao menos duas espécies do gênero Mazama [M. americana (Erxleben, 1777) e M. gouazoubira (G. Fischer, 1814)], mas preferiuse agrupar os registros dessas espécies de Mazama em nível de gênero para afastar a possibilidade de erro de identificação em campo.

Revista Árvore, Viçosa-MG, v.36, n.6, p.1061-1072, 2012 
Tabela 1 - Esforços de amostragem realizada em transectos na região do Cristalino, estado do Mato Grosso, Brasil. $\mathrm{N}$ = número de levantamentos (censos em transecções lineares) e $\mathrm{L}=$ distância percorrida.

Table 1 - Effort sampling transects conducted in the Cristalino region, Mato Grosso state, Brazil. $N=$ number of surveys (censuses in linear transects) and $L=$ distance traveled.

\begin{tabular}{clcccr}
\hline \multirow{2}{*}{ Esforço deamostragem } & \multicolumn{2}{l}{ Ambientes sem turismo } & \multicolumn{2}{c}{ Ambientes com turismo } & \multirow{2}{*}{ Total (Km) } \\
\cline { 2 - 5 } & $\mathrm{N}$ & $\mathrm{L}(\mathrm{Km})$ & $\mathrm{N}$ & $\mathrm{L}(\mathrm{Km})$ & \\
\hline Diurno & 68 & 183,3 & 93 & 178,0 & 361,3 \\
Noturno & 22 & 69,4 & 19 & 37,6 & 107,0 \\
\hline Total & 90 & 252,7 & 112 & 215,6 & 468,3 \\
\hline
\end{tabular}

\subsection{Análise dos dados}

A partir dos dados coletados nos transectos e nas parcelas, estimativas de riqueza de espécies, cálculo de similaridade e comparações da abundância das espécies foram realizados. Dados adicionais obtidos fora dos sítios dos transectos foram utilizados apenas para complementar o inventário de espécies de mamíferos da região, não sendo considerados nas análises estatísticas.

\subsubsection{Riqueza e similaridade de espécies}

A riqueza de espécies de mamíferos de cada tipo de ambiente foi estimada pelo estimador Jackknife 1 (HELTSHE e FORRESTER, 1983), utilizando o Programa EstimateS versão 8.2 (COLWELL, 2009). Nessa análise, adotou-se como unidade amostral a frequência de registro (direto e indireto) das espécies por dia de trabalho em campo para cada tipo de ambiente. A estimativa de riqueza de espécies é apresentada seguida de seu intervalo de confiança (IC), em nível de significância de $95 \%(\mathrm{p}<0,05)$.

A semelhança faunística entre os dois tipos ambientes amostrados foi analisada utilizando o índice de similaridade de Jaccard (Cj), o qual fornece um valor que varia de 0 a 1 , de forma que, quanto mais próximo de 1 for o $\mathrm{Cj}$, maior será a similaridade entre as áreas comparadas (DURIGAN, 2003).

Adicionalmente, foi processada, utilizando o programa estatístico R (RDEVELOPMENTCORETEAM, 2011), a análise de similaridade ANOSIM para testar se houve diferença significativa na composição específica das amostras obtidas nos ambientes com e sem ecoturismo. Nesta análise, calculou-se o valor de R, que pode apresentar uma amplitude de variação de -1 a 1, e valores próximos de zero indicam que não há diferença significativa entre os ambientes comparados (CLARKE; WARWICK, 2001).

\subsubsection{Comparação da abundância}

Os transectos amostrados apresentaram comprimentos diferentes. Por isso, foi gerado um índice de abundância para padronizar as unidades amostrais e permitir comparação entre a abundância média das espécies. Utilizou-se como índice de abundância a taxa de registro direto de cada espécie por $10 \mathrm{~km}$ percorridos em transectos (CHIARELLO, 1999) em cada tipo de ambiente, a qual foi calculada utilizando a equação: $\mathrm{A}=(\mathrm{N} i / \mathrm{L} i) \times 10$; em que $\mathrm{A}=$ Abundância, $\mathrm{N} i=$ número de registros diretos de cada espécie obtido no levantamento $i$ e L $i=$ distância em Km percorrida no levantamento $i$.

O cálculo da abundância dos mamíferos de hábitos diurnos foi efetuado levando em consideração apenas os levantamentos realizados ao longo do dia. Para aqueles de hábitos noturnos, o cálculo foi conduzido utilizando apenas os levantamentos noturnos. Mas, para os animais que apresentaram atividades diurna e noturna, a análise foi feita somando-se os percursos realizados em levantamentos diurnos e noturnos.

Comparou-se o índice de abundância de cada espécie de mamífero entre os ambientes sem turismo e com ecoturismo, utilizando o teste $\mathrm{t}$, de Student, para amostras independentes (ROCHA et al., 2006).

\section{RESULTADOS}

\subsection{Inventário das espécies}

Obtiveram-se registros de 37 espécies (Tabela II), distribuídas em oito ordens: Carnivora(12 espécies), Primates (sete espécies), Rodentia (seis espécies), Cingulata (quatro espécies), Artiodactyla (três espécies), Pilosa (três espécies), Didelphimorphia (uma espécie) e Perissodactyla (uma espécie).

Revista Árvore, Viçosa-MG, v.36, n.6, p.1061-1072, 2012 
Tabela 2 - Espécies de mamíferos registradas na região do Cristalino, estado do Mato Grosso, Brasil. Legenda do tipo de registro: $\mathrm{Vi}=$ visual $; \mathrm{Vo}=$ vocal $; \mathrm{P}=$ pegadas; $\mathrm{e} \mathrm{T}=$ toca. Os registros considerados como fora dos transectos são aqueles que não ocorreram dentro ou nas proximidades das trilhas

Table 2 - Species of mammals recorded in the Cristalino region, Mato Grosso state, Brazil. Titles of the record type: Vi $=$ visualization $;$ Vo = vocal; $P=$ footprints; and $T=$ burrow. Records outside the transects are those that not occurred within or near the tracks.

\begin{tabular}{|c|c|c|c|}
\hline \multirow{2}{*}{ Taxa } & \multirow{2}{*}{ Nome comum } & \multicolumn{2}{|c|}{ Tipo de registro } \\
\hline & & Sem turismo & Com ecoturismo \\
\hline \multicolumn{4}{|l|}{ Ordem Didelphimorphia } \\
\hline \multicolumn{4}{|l|}{ Família Didelphidae } \\
\hline Didelphis marsupialis Linnaeus, 1758 & Gambá, mucura & $\mathrm{Vi}$ & Vi \\
\hline \multicolumn{4}{|l|}{ Ordem Pilosa } \\
\hline \multicolumn{4}{|l|}{ Família Myrmecophagidae } \\
\hline Myrmecophaga tridactyla Linnaeus, 1758* & Tamanduá-bandeira & $\mathrm{Vi}, \mathrm{P}$ & -- \\
\hline Tamandua tetradactyla (Linnaeus, 1758) & Tamanduá-mirim & $\mathrm{Vi}, \mathrm{P}$ & Vi, P \\
\hline \multicolumn{4}{|l|}{ Família Megalonychidae } \\
\hline Choloepus hoffmanni Peters, 1858 & Preguiça-real & $\mathrm{Vi}$ - fora dos transectos & \\
\hline \multicolumn{4}{|l|}{ Ordem Cingulata } \\
\hline \multicolumn{4}{|l|}{ Família Dasypodidae } \\
\hline Dasypus kappleri Krauss, 1862 & Tatu-15-quilos & $\mathrm{Vi}, \mathrm{P}, \mathrm{T}$ & $\mathrm{Vi}, \mathrm{P}, \mathrm{T}$ \\
\hline Dasypus novemcinctus Linnaeus, 1758 & Tatu-galinha & $\mathrm{Vi}, \mathrm{P}, \mathrm{T}$ & $\mathrm{Vi}, \mathrm{P}, \mathrm{T}$ \\
\hline Priodontes maximus (Kerr, 1792)* & Tatu-canastra & $\mathrm{P}, \mathrm{T}$ & $\mathrm{P}, \mathrm{T}$ \\
\hline Cabassous sp. & Tatu-de-rabo-mole & $\mathrm{T}$ & $\mathrm{P}, \mathrm{T}$ \\
\hline \multicolumn{4}{|l|}{ Ordem Primates } \\
\hline \multicolumn{4}{|l|}{ Família Aotidae } \\
\hline Aotus sp. & Macaco-da-noite & -- & Vi, Vo \\
\hline \multicolumn{4}{|l|}{ Família Atelidae } \\
\hline Ateles marginatus É. Geoffroy, 1809* & Coatá-de-cara-branca & Vi, Vo & Vi, Vo \\
\hline Alouatta belzebul (Linnaeus, 1766) & Bugio-de-mãos-ruivas & Vo & Vi, Vo \\
\hline \multicolumn{4}{|l|}{ Família Cebidae } \\
\hline Cebus apella (Linnaeus, 1758) & Macaco-prego & $\mathrm{Vi}$ & $\mathrm{Vi}$ \\
\hline Mico emiliae (Thomas, 1920) & Sauim, mico & Vi & $\mathrm{Vi}$ \\
\hline \multicolumn{4}{|l|}{ Família Pitheciidae } \\
\hline Callicebus moloch (Hoffmannsegg, 1807) & Zogue-zogue & Vi, Vo & Vi, Vo \\
\hline Chiropotes albinasus (Geoffroy \& Deville, 1848) & Cuxiú & $\mathrm{Vi}$ & $\mathrm{Vi}$ \\
\hline \multicolumn{4}{|l|}{ Ordem Carnivora } \\
\hline \multicolumn{4}{|l|}{ Família Canidae } \\
\hline Cerdocyon thous (Linnaeus, 1766) & Cachorro-do-mato & $\mathrm{P}$ - fora dos transectos & \\
\hline Speothos venaticus (Lund, 1842)* & Cachorro-vinagre & $\mathrm{P}$ & $\mathrm{P}$ \\
\hline \multicolumn{4}{|l|}{ Família Procyonidae } \\
\hline Nasua nasua (Linnaeus, 1766) & Quati & $\mathrm{Vi}$ & $\mathrm{Vi}$ \\
\hline Potos flavus (Schreber, 1774) & Jupará & $\mathrm{Vi}$ & $V_{i}$ \\
\hline \multicolumn{4}{|l|}{ Família Mustelidae } \\
\hline Eira barbara (Linnaeus, 1758) & Irara, papa-mel & $\mathrm{Vi}, \mathrm{P}$ & $\mathrm{Vi}, \mathrm{P}$ \\
\hline Lontra longicaudis (Olfers, 1818) & Lontra & Vi - no rio Cristalino & \\
\hline Pteronura brasiliensis (Gmelin, 1788)* & Ariranha & Vi - no rio Cristalino & \\
\hline \multicolumn{4}{|l|}{ Família Felidae } \\
\hline Panthera onca (Linnaeus, 1758)* & Onça-pintada & $\mathrm{P}$ & $\mathrm{P}$ \\
\hline Puma concolor (Linnaeus, 1771) & Onça-parda & $\mathrm{P}$ & $\mathrm{P}$ \\
\hline Puma yagouaroundi (É. G. Saint-Hilare, 1803) & Gato-mourisco & $\mathrm{P}$ - fora dos transectos & \\
\hline Leopardus pardalis (Linnaeus, 1758)* & Jaguatirica & $\mathrm{P}$ & $\mathrm{P}$ \\
\hline Leopardus wiedii (Schinz, 1821)* \# & Gato-maracajá & $\mathrm{P}$ & $\mathrm{P}$ \\
\hline
\end{tabular}

Revista Árvore, Viçosa-MG, v.36, n.6, p.1061-1072, 2012 
Tabela 2 - Cont.

Table 2 - Cont.

\begin{tabular}{|c|c|c|c|}
\hline \multirow{2}{*}{ Taxa } & \multirow{2}{*}{ Nome comum } & \multicolumn{2}{|c|}{ Tipo de registro } \\
\hline & & Sem turismo & Com ecoturismo \\
\hline \multicolumn{4}{|l|}{ Ordem Perissodactyla } \\
\hline \multicolumn{4}{|l|}{ Família Tapiridae } \\
\hline Tapirus terrestris (Linnaeus, 1758) & Anta & $\mathrm{Vi}, \mathrm{P}$ & $\mathrm{P}$ \\
\hline \multicolumn{4}{|l|}{ Ordem Artiodactyla } \\
\hline \multicolumn{4}{|l|}{ Família Tayassuidae } \\
\hline Pecari tajacu (Linnaeus, 1758) & Cateto, caititu & $\mathrm{Vi}, \mathrm{P}$ & $\mathrm{Vi}, \mathrm{P}$ \\
\hline Tayassu pecari (Link, 1795) & Queixada & Vi, P, Vo & $\mathrm{Vi}, \mathrm{P}, \mathrm{Vo}$ \\
\hline \multicolumn{4}{|l|}{ Família Cervidae } \\
\hline Mazama spp. & Veado & $\mathrm{Vi}, \mathrm{P}$ & $\mathrm{Vi}, \mathrm{P}$ \\
\hline \multicolumn{4}{|l|}{ Ordem Rodentia } \\
\hline \multicolumn{4}{|l|}{ Família Caviidae } \\
\hline Hydrochoerus hydrochaeris (Linnaeus, 1766) & Capivara & -- & $\mathrm{Vi}$ \\
\hline \multicolumn{4}{|l|}{ Família Cuniculidae } \\
\hline Cuniculus paca (Linnaeus, 1766) & Paca & $\mathrm{Vi}, \mathrm{P}$ & $\mathrm{Vi}, \mathrm{P}$ \\
\hline \multicolumn{4}{|l|}{ Família Dasyproctidae } \\
\hline Dasyprocta leporina (Linnaeus, 1766) & Cutia & Vi, Vo, P & Vi, Vo, P \\
\hline \multicolumn{4}{|l|}{ Família Echimyidae } \\
\hline Proechimys sp. & Rato & $\mathrm{Vi}$ & Vi \\
\hline Dactylomys dactylinus (Desmarest, 1817) & Rato-de-bambu & - & Vo \\
\hline \multicolumn{4}{|l|}{ Família Sciuridae } \\
\hline Sciurus aestuans (Linnaeus, 1766) & Caxinganga & $\mathrm{Vi}$ & Vi \\
\hline
\end{tabular}

* Espécies ameaçada de extinção no Brasil (MACHADO et al., 2008);

\# Identificação confirmada com a visualização de 1 indivíduo fora dos transectos.

Oito espécies ameaçadas de extinção no Brasil (MACHADO et al., 2008) foram registradas durante o estudo, sendo seis comuns aos dois tipos de ambientes amostrados: Priodontes maximus (Kerr, 1792), Ateles marginatus É. Geoffroy, 1809, Panthera onca (Linnaeus, 1758), Leopardus pardalis (Linnaeus, 1758), Leopardus wiedii (Schinz, 1821) e Speothos venaticus (Lund, 1842). Pteronura brasiliensis (Gmelin, 1788), uma espécie de hábito semi-aquático, foi registrada no rio Cristalino. Uma única espécie ameaçada de extinção foi exclusiva aos ambientes sem turismo: Myrmecophaga tridactyla Linnaeus, 1758.

Embora a metodologia utilizada se destinasse ao estudo de mamíferos de médio e de grande porte, quatro espécies de mamíferos de pequeno porte foram incluídas na listagem porque puderam ser seguramente identificadas durante a amostragem, sendo elas: Mico emiliae (Thomas, 1920), Proechimys sp., Sciurus aestuans (Linnaeus, 1766) e Dactylomys dactylinus (Desmarest, 1817). Todas elas foram registradas durante a realização de levantamentos em transectos, e as três primeiras apresentaram registros visuais e a última, vocal.
Obteve-se registros de Choloepus hoffmanni Peters, 1858, Lontra longicaudis (Olfers, 1818), P. brasiliensis, Puma yagouaroundi (É. G. Saint-Hilare, 1803) e Cerdocyon thous (Linnaeus, 1766) apenas fora das trilhas amostradas, mas estas espécies foram incorporadas à listagem no intuito de gerar um inventário mais completo para a área de estudo. As três primeiras espécies foram visualizadas durante percursos fluviais no rio Cristalino; $P$. yagouaroundi teve suas pegadas registradas durante percursos aleatórios na RPPN Lote Cristalino e C. thous teve suas pegadas detectadas na estrada que corta o Parque Estadual Cristalino, em locais próximos às fazendas.

\subsection{Estimativa de riqueza e similaridade de espécies}

Nos ambientes sem turismo, a riqueza observada foi de 29 espécies, enquanto a riqueza estimada, de 36 (IC $=5,67)$ espécies. Por sua vez, nos ambientes com ecoturismo foram identificadas 31 espécies, e a riqueza estimada foi de 36 (IC $=4,24)$ espécies.

A curva de acumulação de espécies (curva do coletor) não se estabilizou completamente para os ambientes sem turismo (Figura 2), indicando que novas espécies 
ainda devem ser reveladas. Mas, para os ambientes com ecoturismo a curva do coletor atingiu estabilidade quase completa (Figura 2).

$\mathrm{O}$ índice de similaridade de Jaccard $(\mathrm{Cj})$ foi igual a 0,88, ou seja, os ambientes sem turismo e com ecoturismo apresentaram $88 \%$ de similaridade em espécies. Obteve-se esse valor porque 28 espécies foram comuns aos dois tipos de ambientes estudados; apenas uma espécie foi exclusiva aos ambientes sem turismo e duas ocorreram somente nos ambientes com ecoturismo. De forma semelhante, a análise de ANOSIM mostrou que não houve diferença marcante $(\mathrm{R}=0,051 ; \mathrm{p}=0,001)$ na composição de espécies de mamíferos entre os dois tipos de ambientes comparados, uma vez que o valor para interpretação da similaridade indicou diferença menor que $25 \%(|R|<0,25)$.

\subsection{Abundância de mamíferos em transectos}

O índice de abundância dos mamíferos, dado pelo número médio de registros diretos de cada espécie por $10 \mathrm{~km}$ percorridos em transecções lineares, foi comparado a fim de verificar a existência de diferença estatisticamente significativa na abundância das espécies entre os ambientes sem turismo e com ecoturismo. Entre os 25 taxa comuns aos dois tipos de ambientes, 21 não apresentaram diferenças

Sem ecoturismo

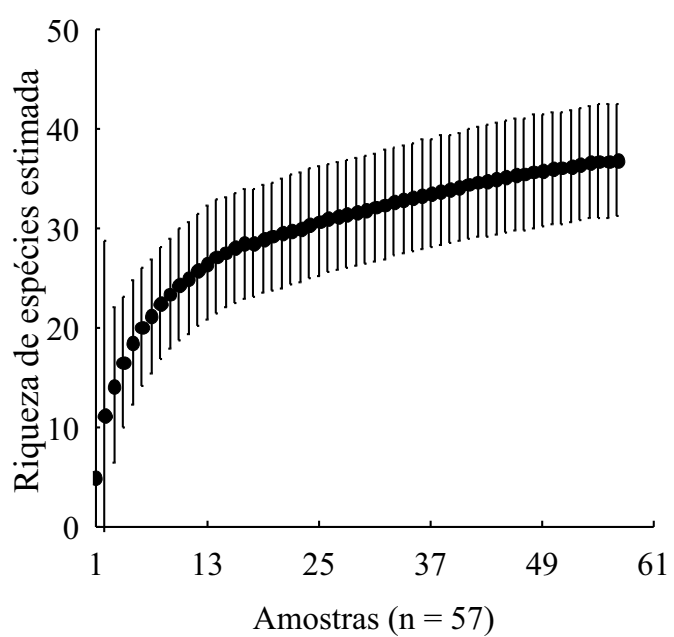

significativas entre seus índices de abundância (teste t de Student; $\mathrm{p}<0,05)$ e quatro mostraram índices de abundância significativamente diferentes: Dasyprocta leporina (Linnaeus, 1766), Mazama spp. e Dasypus kappleri Krauss, 1862 foram mais abundantes nos ambientes sem turismo; já $M$. emiliae apresentou índice de abundância maior nos ambientes com turismo (Tabela 3).

Os índices de abundância obtidos para $A$. marginatus, a espécie símbolo do Parque Estadual Cristalino, nos ambientes sem turismo (0,72 grupo/ $10 \mathrm{~km}$ percorridos) e nos ambientes com ecoturismo (0,86 grupo/10 $\mathrm{km}$ percorridos), não diferiram estatisticamente.

\section{DISCUSSÃO}

A riqueza de mamíferos registrada (37 espécies) na área de estudo é considerada elevada e inclui espécies potenciais indicadoras de boa qualidade dos ambientes, como A. marginatus e D. kappleri. Embora a riqueza de espécies observada nos ambientes com ecoturismo tenha se mostrado ligeiramente maior que nos ambientes sem turismo, a riqueza estimada foi semelhante entre os dois ambientes estudados. Além disso, a composição de espécies foi bastante similar entre os ambientes com e sem ecoturismo.

Com ecoturismo

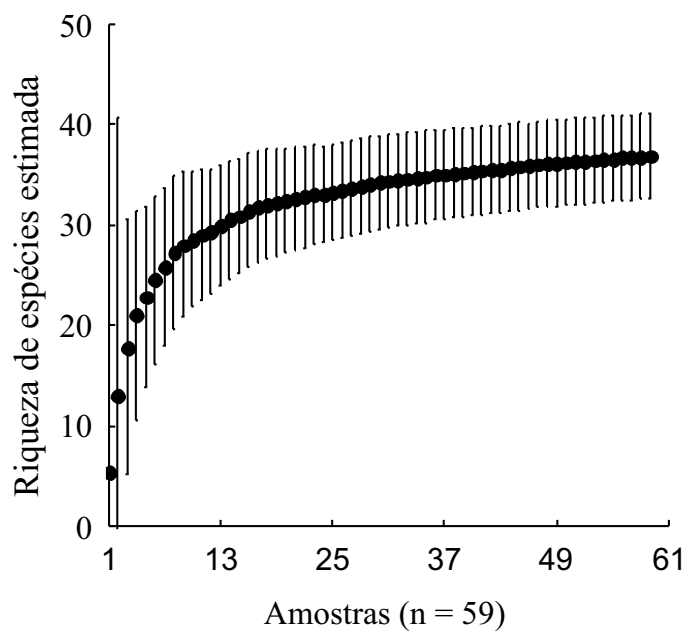

Figura 2 - Curvas do coletor construídas a partir da riqueza de espécies de mamíferos estimada (Jackknife 1) para a região do Cristalino, estado do Mato Grosso, Brasil. Os pontos indicam os valores médios da riqueza estimada e as barras fornecem o intervalo de confiança, $\mathrm{p}<0,05$.

Figure 2 -Rarefaction curves prepared with the species richness estimated of mammals, with the Jackknife 1 estimator, for the Cristalino region, Mato Grosso state, Brazil. The dots indicate the average values of richness and bars provide the confidence interval $(p<0.05)$.

Revista Árvore, Viçosa-MG, v.36, n.6, p.1061-1072, 2012 
Tabela 3 - Comparação da abundância das espécies de mamíferos registradas na região do Cristalino, estado do Mato Grosso, Brasil, dada pelo número médio de registros diretos de cada espécie por $10 \mathrm{~km}$ percorridos em transectos. Os valores da probabilidade $(\mathrm{P})$ em negrito indicam diferenças significativas na abundância pelo teste t de Student $(\mathrm{p}<0,05)$.

Table 3 - Comparison of the abundance of mammal species recorded in the Cristalino region, Mato Grosso state, Brazil, given by the average number of direct record of each species per $10 \mathrm{~km}$ traveled in transects. The values of probability $(P)$ in bold indicate significant differences in abundance by Student's t test $(p<0.05)$.

\begin{tabular}{|c|c|c|c|c|c|}
\hline \multirow{2}{*}{ Espécies } & \multirow{2}{*}{ Período de atividade } & \multicolumn{2}{|c|}{ Abundância ( \pm desvio padrão) } & \multirow{2}{*}{ t-valor } & \multirow{2}{*}{$P$} \\
\hline & & Sem turismo & Com ecoturismo & & \\
\hline Dasyprocta leporina & Diurno e noturno & $2,43 \pm 4,11$ & $0,92 \pm 2,65$ & $-3,154$ & $\mathbf{0 , 0 0 2}$ \\
\hline Cebus apella & Diurno & $2,35 \pm 2,79$ & $2,63 \pm 3,23$ & $-0,591$ & 0,555 \\
\hline Mazama spp. & Diurno e noturno & $1,74 \pm 2,53$ & $0,91 \pm 2,08$ & $-2,559$ & $\mathbf{0 , 0 1 1}$ \\
\hline Potos flavus & Noturno & $1,74 \pm 2,33$ & $1,18 \pm 2,45$ & $-0,746$ & 0,460 \\
\hline Chiropotes albinasus & Diurno & $1,01 \pm 2,35$ & $0,48 \pm 1,59$ & 1,711 & 0,089 \\
\hline Cuniculus paca & Noturno & $0,85 \pm 1,73$ & $2,47 \pm 3,67$ & 1,842 & 0,073 \\
\hline Ateles marginatus & Diurno & $0,72 \pm 1,70$ & $0,86 \pm 2,13$ & $-0,419$ & 0,676 \\
\hline Dasypus kappleri & Diurno e noturno & $0,45 \pm 1,30$ & $0,04 \pm 0,40$ & $-3,199$ & 0,002 \\
\hline Proechimys sp. & Noturno & $0,29 \pm 0,93$ & $0,82 \pm 1,97$ & 1,122 & 0,269 \\
\hline Callicebus moloch & Diurno & $0,27 \pm 0,97$ & $0,50 \pm 1,61$ & $-1,053$ & 0,294 \\
\hline Mico emiliae & Diurno & $0,19 \pm 0,79$ & $0,68 \pm 1,82$ & $-2,073$ & 0,040 \\
\hline Didelphis marsupialis & Noturno & $0,14 \pm 0,68$ & $0,28 \pm 1,23$ & 0,455 & 0,652 \\
\hline Sciurus aestuans & Diurno & $0,11 \pm 0,62$ & $0,04 \pm 0,39$ & 0,827 & 0,409 \\
\hline Pecari tajacu & Diurno & $0,09 \pm 0,52$ & $0,09 \pm 0,64$ & $-0,038$ & 0,970 \\
\hline Dasypus novemcinctus & Diurno e noturno & $0,07 \pm 0,62$ & $0,23 \pm 0,97$ & 1,378 & 0,170 \\
\hline Tamandua tetradactyla & Diurno e noturno & $0,07 \pm 0,46$ & $0,19 \pm 0,99$ & 1,031 & 0,304 \\
\hline Nasua nasua & Diurno & $0,05 \pm 0,38$ & $0,00 \pm 0,00$ & -- & -- \\
\hline Tayassu pecari & Diurno & $0,05 \pm 0,43$ & $0,07 \pm 0,65$ & $-0,171$ & 0,865 \\
\hline Alouatta belzebul & Diurno & $0,05 \pm 0,43$ & $0,05 \pm 0,52$ & $-0,021$ & 0,983 \\
\hline Myrmecophaga tridactyla & Diurno e noturno & $0,04 \pm 0,33$ & $0,00 \pm 0,00$ & $-1,116$ & 0,266 \\
\hline Tapirus terrestris & Diurno e noturno & $0,04 \pm 0,33$ & $0,00 \pm 0,00$ & $-1,116$ & 0,266 \\
\hline Dactylomys dactylinus & Noturno & $0,00 \pm 0,00$ & $0,72 \pm 2,18$ & - & -- \\
\hline Aotus sp. & Noturno & $0,00 \pm 0,00$ & $0,62 \pm 1,93$ & - & - \\
\hline Eira barbara & Diurno & $0,00 \pm 0,00$ & $0,04 \pm 0,43$ & - & - \\
\hline Hydrochoerus hydrochaeris & Diurno e noturno & $0,00 \pm 0,00$ & $0,04 \pm 0,40$ & -- & - \\
\hline
\end{tabular}

Considerando que os dois tipos de ambientes estudados são relativamente próximos e apresentavam a estrutura da vegetação equivalente, esperavase que a similaridade fosse alta e a riqueza de espécies não fosse estatisticamente diferente caso as atividades de ecoturismo desenvolvidas estivessem exercendo pouco efeito adverso sobre a permanência das espécies. Tal fato foi confirmado neste estudo.

A alta similaridade e a semelhança na riqueza de espécies entre os ambientes estudados são reflexos do tipo de empreendimento considerado e do perfil dos turistas que visitaram a área, os quais percorreram as trilhas acompanhados de guias, em grupos com número reduzido de pessoas, produzindo relativamente pouco ruído, com os objetivos de observar e contemplar a natureza, incluindo a observação de animais, principalmente aves. Ademais, o grau de utilização da área parece não superar a sua capacidade de suporte.

As abundâncias de D. leporina e de Mazama spp., menores nos ambientes com ecoturismo, podem ter sido causadas pela movimentação de pessoas nas trilhas que, mesmo se deslocando em grupos pequenos e com baixo nível de ruído, acabam afugentando os animais das proximidades das trilhas. O efeito cumulativo desses afugentamentos provavelmente induz os animais a evitarem as proximidades das trilhas, tornando-os menos abundantes nesses locais. Durante a coleta de dados, os encontros com indivíduos de D. leporina e Mazama spp. foram sempre seguidos por afastamentos dos animais das proximidades das trilhas. 
O índice de abundância de D. leporina e de Mazama spp., mesmo considerando apenas os valores observados nos ambientes com ecoturismo, leva a situá-los entre os mamíferos mais abundantes da área de estudo (Tabela 3). Esse fato permite inferir que os impactos das atividades de ecoturismo desenvolvidas na área e experimentados por essas espécies foram de pequena magnitude, uma vez que seus índices de abundância permaneceram altos, embora menores que nos ambientes sem turismo.

O tatu Dasypus kappleri, embora tenha período de atividade predominantemente noturno, teve sua abundância negativamente afetada pelas atividades antrópicas, as quais são desenvolvidas principalmente ao longo do dia. Pois D. kappleri parece ser uma espécie indicadora da qualidade ambiental, sendo bastante sensível a perturbações no ambiente e deve realmente estar reduzindo suas atividades nos sítios com utilização antrópica.

Como medidas mitigadoras indicadas para reduzir os impactos ambientais negativos sobre as espécies de mamíferos, sugerem-se: 1- garantir que o zoneamento da área deixe como zona intangível e, ou, primitiva e, ou, silvestre, conforme a categoria da unidade de conservação considerada, uma porção significativa de hábitats contínuos adequados para a ocorrência das espécies adversamente afetadas; 2 - manter a visitação abaixo da capacidade de suporte do ambiente, uma vez que o sucesso do empreendimento está diretamente relacionado com a manutenção da boa qualidade ambiental, de forma que, se as espécies de interesse turístico deixarem de ocorrer na área, o turista perderá o interesse em visitá-la. Por isso, o porte do empreendimento e a intensidade de uso da área sempre devem respeitar a capacidade de suporte do ambiente; 3- conduzir a visitação em grupos com número reduzido de pessoas, as quais devem ser acompanhadas por guias treinados, buscando realizar os percursos produzindo baixo nível de ruído e com o mínimo de alteração no ambiente; 4- coibir a realização de caça e de desmatamentos clandestinos; e 5- impedir a entrada de animais domésticos, especialmente cães e gatos, na unidade de conservação. Essas medidas mitigadoras já estavam sendo satisfatoriamente adotadas nas áreas das RPPNs, mas ainda precisavam ser implantadas dentro dos limites do Parque Estadual Cristalino.

Vale destacar que as atividades de ecoturismo precisam ser desenvolvidas sem a disponibilização de qualquer atrativo alimentar aos animais, uma vez que esses atrativos podem aumentar a dependência e aproximação dos animais silvestres com os seres humanos. Além disso, tal comportamento pode condicionar os animais a relacionarem a presença dos humanos com a disponibilidade de alimento, o que pode ser perigoso quando se trata de animais com potencial para predar seres humanos, como é o caso da $P$. onca.

Os valores obtidos para a abundância do primata A. marginatus indicam que ele não evitou os sítios utilizados em atividades de ecoturismo e posicionam-no entre as espécies de mamíferos com abundância intermediária na região do Cristalino (Tabela 3), mostrando que essa espécie é comum na área de estudo e tornando evidente a importância dessa região para sua conservação. Em termos comparativos, um estudo realizado por Ravetta e Ferrari (2009) na região do baixo rio Tapajós, Amazônia Central brasileira, apontou que A. marginatus esteve presente em apenas cinco das 14 áreas amostradas e que essa espécie estava extinta em fragmentos pequenos (<100 ha).

A espécie Myrmecophaga tridactyla foi registrada apenas duas vezes e nos ambientes sem turismo, indicando que ela é rara na área de estudo e a inexistência de registros nos ambientes com turismo pode não refletir apenas o efeito adverso da atividade antrópica, mas também a dificuldade para se detectarem espécies raras. Entretanto, a falta de registros de Aotus sp. nos ambientes sem turismo, onde o esforço de amostragem noturna foi maior e a ocorrência da espécie era esperada, não é compreendida. É provável que a intensificação do esforço de amostragem noturna acabe por registrála também nos ambientes sem turismo.

O canídeo Cerdocyon thous parece associado somente a áreas abertas para pastagem de gado no Parque, não sendo encontrado no interior da floresta primária. Aparentemente, a colonização de novas áreas por essa espécie depende da abertura de estradas e, ou, picadões que liguem as áreas colonizadas e não colonizadas, haja vista que essa espécie utiliza preferencialmente ambientes com vegetação aberta (TROVATI et al., 2007).

Os resultados deste estudo corroboram, em termos de riqueza e abundância de mamíferos de médio e de grande porte, a afirmação apresentada por Magro (2003) de que o ecoturismo, quando bem manejado, tem grande potencial de gerar empregos e benefícios econômicos, com menor prejuízo ao meio ambiente.

Revista Árvore, Viçosa-MG, v.36, n.6, p.1061-1072, 2012 


\section{CONCLUSÕES}

Registros de 37 espécies de mamíferos simpátricas foram obtidos durante este estudo, riqueza que pode ser considerada elevada. Além disso, a composição taxonômica apresentada relaciona espécies de mamíferos indicadores potenciais de boa "saúde" dos ambientes amostrados na região do Cristalino, como A. marginatus e D. kappleri.

A riqueza de espécies, observada e estimada, não apresentou diferença estatisticamente significativa entre os ambientes com ecoturismo e sem turismo. Tal fato, aliado à alta similaridade de espécies encontrada entre os ambientes amostrados, permite concluir que o grau de utilização antrópica não causou efeito adverso significativo sobre a permanência das espécies de mamíferos de médio e de grande porte nos ambientes utilizados em atividades de ecoturismo.

No que se refere à abundância das espécies, entre os 25 taxa comuns aos dois tipos de ambientes amostrados, apenas três apresentaram índices de abundância estatisticamente inferiores nos ambientes com ecoturismo, sendo eles: D. leporina, Mazama spp. e D. kappleri.

Percebe-se, portanto, que o impacto das atividades de ecoturismo desenvolvidas durante o estudo teve pequena magnitude, em termos de riqueza e abundância das espécies de mamíferos, e que esse tipo de empreendimento se apresenta como importante atividade econômica a ser desenvolvida em áreas com potencial ecoturístico na Amazônia, à medida que concilia a conservação dos recursos naturais e a geração de renda. Mas é importante monitorar os recursos ambientais e adotar medidas ambientais mitigadoras para reduzir os impactos ambientais negativos.

\section{AGRADECIMENTOS}

Às instituições e às pessoas que contribuíram para viabilizar a realização deste estudo; ao CNPq, pelas bolsas de doutorado e de produtividade em pesquisa concedidas ao primeiro e ao segundo autor, respectivamente; à Sra. Vitória da Riva Carvalho, por autorizar a realização da pesquisa nas RPPNs Cristalino e Lote Cristalino e por todo o suporte logístico; ao pessoal da Fundação Ecológica Cristalino e do Hotel de Selva Cristalino, pela colaboração e suporte logístico; aos docentes Sebastião Venâncio Martins e Guido Assunção Ribeiro, pela leitura crítica e pelas sugestões no manuscrito; ao Sr. Leandro Juen, pelo auxílio nas análises estatísticas; e aos pesquisadores José de Sousa e Silva Jr. e Gil Iack Ximenes, pela cordial ajuda na identificação de duas espécies de mamíferos.

\section{REFERÊNCIAS}

BRASIL. Ministério do Meio Ambiente. Áreas prioritárias para a conservação, uso sustentável e repartição de benefícios da biodiversidade brasileira: atualização - Portaria MMA n ${ }^{\circ}$ 09, de 23 de janeiro de 2007. Brasília: 2007. 300p.

BUCKLAND, S. T. et al. distance sampling. estimating abundance of biological populations. London: Chapman \& Hall, 1993. 446p.

CHIARELLO, A. C. Effects of fragmentation of the Atlantic forest on mammal communities in southeastern Brazil. Biological Conservation, v.89, n.1, p.71-82, 1999.

CHIARELLO, A. G. Density and population size of mammals in remnants of Brazilian Atlantic Forest. Conservation Biology, v. 14, n.6, p.1649-1657, 2000.

CLARKE, K. R.; WARWICK, R. M. Change in marine communities: an approach to statistical analysis and interpretation. Plymouth: Natural Environment Research Council, 2001. $172 \mathrm{p}$.

COLWELL, R. K. EstimateS: statistical estimation of species richness and shared species from samples. Version 8.2. 2009. Disponível em: $<$ http://viceroy.eeb.uconn.edu/EstimateS $>$. Acesso em: 24 mar. 2010.

COSTA, L. P. et al. Mammal conservation in Brazil. Conservation Biology, v.19, n.3, p.672-679, 2005.

CULLEN JR, L.; RUDRAN, R. Transectos lineares na estimativa de densidade de mamíferos e aves de médio e grande porte. In: CULLEN JR, L.; RUDRAN, R.; VALLADARES-PÁDUA, C. (Orgs). Métodos de estudos em biologia da conservação e manejo da vida silvestre. Curitiba: Universidade Federal do Paraná, 2003. p.169-179.

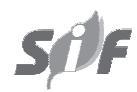

Revista Árvore, Viçosa-MG, v.36, n.6, p.1061-1072, 2012 
DURIGAN, G. Métodos para análise de vegetação arbórea. In: CULLEN JR, L.; RUDRAN, R.; VALLADARES-PÁDUA, C., (Org). Métodos de estudos em biologia da conservação e manejo da vida silvestre. Curitiba:

Universidade Federal do Paraná, 2003. p.455-479.

FUNDAÇÃO ESTADUAL DO MEIO AMBIENTE FEMA. Parque Estadual Cristalino: um lugar para se conservar. Cuiabá: 2002. 20p.

HELTSHE, J. F.; FORRESTER, N. E. Estimating species richness using the Jackknife procedure. Biometrics, v.39, n.1, p.1-11, 1983.

LOBO, A. C.; SIMÕES, L.L. (Org.). Manual de monitoramento e gestão dos impactos da visitação em unidades de conservação. São Paulo: Secretaria de Meio Ambiente/WWF, 2010. 78p.

MACHADO, A. B. M.; DRUMMOND,G. M.; PAGLIA,A. P. (Ed.). Livro vermelho da fauna brasileira ameaçada de extinção. Brasília: Ministério do Meio Ambiente, 2008. 1420p.

MAGRO, T. C. Percepções do uso público em UCs de proteção integral. In: BARGER, A. (Org.). Áreas protegidas: conservação no âmbito do cone sul. Pelotas: Alex Barger, 2003. p.87-98.

MICHALSKI, F.; PERES, C. A. Disturbance-mediated mammal persistence and abundance-area relationships in Amazonian Forest fragments. Conservation Biology, v.21, n.6, p.1626-1640, 2007.

NIMER, E. Clima. In: INSTITUTO BRASILEIRO DE GEOGRAFIA E ESTATÍSTICA - IBGE. Geografia do Brasil - Região CentroOeste. Rio de Janeiro: 1989. v.1. p.23-34.

PAGLIA, A. P. et al. Lista anotada dos mamíferos do Brasil. 2.ed. Belo Horizonte: Conservation International, 2012. 76p. (Occasional paper, 6)

PRADO, M. R.; ROCHA, E. C.; GIÚDICE, G. M. L. D. Mamíferos de médio e grande porte em um fragmento de Mata Atlântica, Minas Gerais, Brasil. Revista Árvore, v.32, n.4, p.741-749, 2008.

R DEVELOPMENT CORE TEAM. R: A language and environment for statistical computing. Version 2.14, 2011. Disponível em: <http://www.R-project.org/>. Acesso em: 10 nov. 2011.
RAVETTA, A. L.; FERRARI, S. F. Geographic distribution and population characteristics of the endangered white-fronted spider monkey (Ateles marginatus) on the lower Tapajós River in central Brazilian Amazonia. Primates, v.50, n.3, p.261-268, 2009.

REIS, N. R.; PERACCHI, A. L.; ANDRADE, F. R. (Org.), Primatas brasileiros. Londrina: Technical Books, 2008. 259p.

ROCHA, E.C. et al. Evaluación estacional de la riqueza y abundancia de especies de mamíferos en la Reserva Biológica Municipal "Mário Viana", Mato Grosso, Brasil. Revista de Biología Tropical, v.54, n.3. p.879-888, 2006.

ROCHA, E. C.; SILVA, E. Composição da mastofauna de médio e grande porte na Reserva Indígena "Parabubure", Mato Grosso, Brasil. Revista Árvore, v.33, n.3, p.451-459, 2009.

SECRETARIA DE ESTADO DE PLANEJAMENTO E COORDENAÇÃO GERAL - SEPLAN. Distribuição da pluviosidade média anual (1983-1994). Zoneamento Sócioeconômico Ecológico. PRODEAGRO, 2001. Disponível em: <http:// www.zsee.seplan.mt.gov.br/mapaspdf/>. Acesso em: 02 jan. 2010.

SILVA, J. M. C.; RYLANDS, A. B.; FONSECA, G. A. B. The fate of the Amazonian areas of endemism. Conservation Biology, v.19, n.3, p.689-694, 2005.

TROVATI, R. G.; BRITO, B. A.; DUARTE, J. M. B. Área de uso e utilização de habitat de cachorrodo-mato (Cerdocyon thous linnaeus, 1766) no cerrado da região central do Tocantins, Brasil. Mastozoología Neotropical, v.14, n.1, p.61-68, 2007.

WILSON, D. E.; REEDER, D. A. (Ed.). Mammal species of the world: a taxonomic and geographic reference. 3.ed. Baltimore: The Johns Hopkins University Press, 2005. 2142p.

ZAPPI, D. C. et al. Plantas vasculares da região do Parque Estadual Cristalino, norte de Mato Grosso, Brasil. Acta Amazonica, v.41, n.1, p.29-38, 2011. 